DOI: https://doi.org/10.46296/gt.v4i7.0021

\title{
NIVELES DE ESTRÉS EN MILITARES EN SERVICIO PASIVO DE LA ASOCIACIÓN DE MILITARES EN SERVICIO PASIVO DE LAS FF.AA. "VETERANOS DE GUERRA Y ANEXOS" DE SANTO DOMINGO DE LOS TSÁCHILAS, DURANTE EL PERIODO DEL 2020-2021
}

\section{STRESS LEVELS IN MILITARY IN PASSIVE SERVICE FROM THE ASSOCIATION OF MILITARY IN PASSIVE SERVICE OF THE ARMED. "VETERANS OF WAR AND ANNEXES" OF SANTO DOMINGO DE LOS TSÁCHILAS, DURING THE PERIOD OF 2020-2021}

\author{
Cedeño-Cedeño Hernán ${ }^{1 *}$; Ordóñez-Tixe Lenín ${ }^{2}$ \\ ${ }^{1}$ Magíster en Psicología Clínica, Psicólogo Clínico, Docente de Intervención \\ Profesional de la Universidad Técnica de Manabí. Portoviejo, Ecuador. \\ ${ }^{2}$ Facultad de Ciencias Humanísticas, Universidad Técnica de Manabí. Portoviejo, \\ Ecuador. https://orcid.org/0000-0001-8171-976X
}

*Correo: hernanandrescedeno@gmail.com

\begin{abstract}
Resumen
En la profesión de militar se está en un constante contacto con diversos factores estresantes, por el mismo hecho de pertenecer a una organización que busca la rigidez, adoctrinamiento y obediencia al momento de realizar diversas actividades, más aún, cuando los militares participan en guerra, como es el caso de la población estudiada que participaron en la Guerra del CENEPA, deben afrontar y resolver por si mismos el estrés desarrollado, el mismo que produce afectaciones biopsicosociales. Para esta investigación se utilizó el inventario de estrés de Melgosa, en el mismo que participaron 87 militares en servicio pasivo. Podemos apreciar que en los militares de servicio pasivo mayores de 60 años de edad presentan en su mayor parte un estrés bajo, por otro lado, los que tienen menos de 60 años de edad presentan en mayor medida un estrés normal. Respondiendo a los resultados, se puede concluir que se percibe en la población mayor de 60 años de edad un resalte del $48 \%$ se encuentra en la zona 2 que representa un nivel bajo de estrés, mientras que los sujetos menores a 60 años de edad sobresale en la zona 3 denominando un nivel normal de estrés, con un 54\% de la población, es decir, de manera general no se percibe un estrés en gran medida, sin embargo, se debe tener presente que en un menor porcentaje existen militares que necesitan de un profesional de la salud mental, para poder continuar en su vida laboral con un buen rendimiento e incluso que no se vea afectado ningún parámetro de su vida.
\end{abstract}

Palabras clave: militares, estrés, salud mental, investigación, psicología.

\begin{abstract}
In the military profession, one is in constant contact with various stressors, due to the very fact of belonging to an organization that seeks rigidity, indoctrination and obedience when carrying out various activities, even more so, when the military participates in war, As is the case of the studied population that participated in the CENEPA War, they must face and solve the stress developed by themselves, the same one that produces biopsychosocial affectations. For this research, the Melgosa stress inventory was used, in which 87 soldiers in passive service participated. We can see that in the passive service military over 60 years of age, most of them present low stress, on the other hand, those who are less than 60 years of age present to a greater extent normal stress. Responding to the results, it can be concluded that in the population over 60 years of age a
\end{abstract}

Información del manuscrito:

Fecha de recepción: 16 de noviembre de 2020

Fecha de aceptación: 04 de enero de 2021

Fecha de publicación: 11 de enero de 2021. 
highlight of $48 \%$ is found in zone 2 , which represents a low level of stress, while the subjects under 60 years of age stand out in zone 3 calling a normal level of stress, with $54 \%$ of the population, that is to say, in general, stress is not perceived to a great extent, however, it should be borne in mind that in a smaller percentage there are soldiers who need from a mental health professional, to be able to continue in your working life with a good performance and even that no parameter of your life is affected.

Keywords: military, stress, mental health, research, psychology.

\section{Introducción}

Dentro del ámbito del servicio militar se debe comprender que se está hablando de personas que se encuentran en un entorno complejo que a su vez es de alguna manera, unilateral, con esto nos referimos que existe una estructura jerárquica inquebrantable, que está establecida por valores y normas éticas que guían el comportamiento del sujeto, el realizar una actividad dictada por otro superior, teniendo en cuenta que la vida militar moldea a la persona, de alguna manera consciente 0 inconsciente se produce un condicionamiento. Rodrigo (2015) da una breve explicación sobre lo que es ser sexo masculino, es "...entendido como lo dado biológico...", por lo tanto, es como nace cada persona biológicamente, sea masculino 0 femenino.

Domjan (2016) nos explica que "las condiciones en las que el aprendizaje puede tener lugar, qué tanto persisten las respuestas aprendidas, y si las circunstancias en que se tiene acceso a la información aprendida la hacen accesible o no" (p.12), a todo este aprendizaje se debe añadir que los sujetos que son parte de esta investigación son ex combatientes del CENEPA, por lo tanto, es una vida llevada con un régimen estricto y con constantes factores estresantes, entendido ese punto se comprenderá que estas personas una vez cumplido este periodo de vida, son de alguna manera expuestos a una realidad/vida que no conocían, establecida por un estilo un tanto diferente, sin tantas restricciones y/o cumplimientos estrictos, donde existe en cierta manera una libertad en cuanto a las acciones $y$ actividades a realizar.

En el boletín informativo que se titula la cohesión: el elemento humano en el combate (tercera parte), es de 
gran importancia tener el control sobre los soldados, esto solo se llevará mediante valores y códigos comportamentales que los mantengan fieles a la unidad en medio del combate (Personal, reclutamiento y movilización, 1987).

En el Centro de Instrucción de Medicina Aeroespacial de Madrid se realizó un estudio nos da a conocer que las respuestas de estrés en el campo de la aviación civil como militar eran de aproximadamente de una cuarta parte de los casos registrados, con estos datos se puede entender a más que la salud mental de estas personas está en riesgo, puede ser un potencial desencadenante para diversos trastornos que pueden ser psiquiátricos como no psiquiátricos. Algo muy importante que comentan Rodríguez y Arce (2016) es sobre los soldados que son enviados a misiones internacionales "debe pasar un reconocimiento psicológico previo, así como recibir formación sobre medidas para favorecer su adaptación psicológica al nuevo contexto de trabajo" y además, da mención a los distintos factores estresantes como "las largas e intensivas jornadas laborales, los problemas de sueño, los problemas relacionales, la separación del núcleo familiar o la exposición a situaciones traumáticas", esto va más allá de la afectación en su desempeño como soldado, si no es evaluado y tratado se producirá serias afectaciones en la salud mental de nuestros compatriotas.

Teniendo en cuenta a De Dios Duarte, Varela, Braschi, y Sánchez (2017) en otro apartado son más específicos en cuento a lo que afecta en el estrés laboral, contenido del puesto, grado de responsabilidad, conflicto y ambigüedad de roles, contacto social y clima organizacional, carga de trabajo, necesidades de mantenimiento $y$ desarrollo de las calificaciones profesionales, horarios y turnos irregulares.

Ospina (2016) revela algo que tendrá gran impacto en la forma de ver e interpretar el estrés, el estrés moderado ayuda a que se produzca un funcionamiento correcto ante diferentes circunstancias, al momento de existir un estrés persistente causa un malestar en el individuo, lo que recalca en gran medida es que el estrés es una respuesta a estímulos internos 0 
externos. Medina en el 2017 da a conocer que el estrés se producirá dependiendo de gran variedad de factores, como la edad, el sexo, la sociedad, la profesión, el tiempo que se lleve haciendo dicha profesión, conflictos de valores, el control que le establezca el trabajo, experiencia, etc.

En la investigación realizada por Acurio (2017), nos presenta como conclusión que el Grupo de Caballería No. 32 "Azuay" de la Brigada de Caballería Blindada No. 11 "Galápagos", el $52 \%$ de los sujetos presentaron altos niveles de estrés laboral, el 36\% presentaron bajo nivel de estrés laboral $\mathrm{y}$, por último, el $12 \%$ presento un nivel de estrés intermedio. Flores (2019) nos indica que, en Ecuador, en el Ministerio de Trabajo, específicamente en la Dirección de Seguridad y Salud, trabaja en conjunto con instituciones tanto privadas como públicas en formación de programas para reducir el riesgo laboral, mejorar las condiciones de trabajo y sobre todo salvaguardar la salud y bienestar de los trabajadores.

Para esta investigación se basó en el concepto que nos indica Melgosa
(1995), este autor desarrolló el instrumento que se utilizó en este estudio, definiéndola como "un conjunto de reacciones fisiológicas y psicológicas que experimenta el organismo cuando se lo somete a fuertes demandas". A menudo el autor considera que el estrés es un potenciador de las enfermedades, aunque no es la causa directa de los mismos. Según este autor el estrés se centra en dos componentes básicos: los agentes estresantes 0 estresores, que son las circunstancias del entorno que lo producen y las respuestas al estrés, en las respuestas del estrés que son las reacciones del individuo antes los mencionados agentes.

\section{Revisión teórica}

El tema del estrés va cobrando mayor importancia en diferentes ámbitos, esto se debe a la relación que existe entre la gran interacción existente entre el ser humano y los distintos factores que surgen diariamente. Cada día se busca fomentar la importancia de un correcto desarrollo de salud mental, interpretándose como: utilización de distintas estrategias que ayuden a la relajación, conocer nuevas técnicas 
de resolución de problemas, contar con un grupo de apoyo, es decir, aprovechar eficazmente los recursos existentes y así poder mejorar la calidad de vida.

El abordaje de la psicología en el ámbito militar es suscitada por los conflictos tanto internos como externos que suelen presentarse en los miembros de las fuerzas armadas, Ruiz (2017) nos reitera que el estrés es un trastorno de gran relevancia, debe ser estudiando y analizado, porque como ya sabemos cada acontecimiento puede ser interpretado de diferente manera, según la persona, puede producir alguna afectación en alguna esfera psicológica o por otro lado la persona puede superar el hecho sin mayor complicación. Teniendo en cuenta que después de estar gran parte de su vida en el ámbito militar, de haber moldeado su comportamiento, forma de pensar y su medio social, deban de salir al mundo una vez jubilados.

Según los conocimientos aportados por Espinoza, Pernas y Gonzales (2018) sobre el estrés, comentan que "es una condición psicológica y física que altera el estado normal del cuerpo, generan afecciones 0 cambios notorios en la persona, cuyos efectos inciden en la salud física y mental, en el rendimiento laboral y académico, provocando preocupación y angustia" (p. 700).

En el artículo expuesto por De Dios Duarte, Varela, Braschi y Sánchez (2017) nos explica que las situaciones que para una persona son estresantes, no es necesario estresante para el resto de la población, no todos perciben circunstancias de la misma manera, por lo tanto, debe analizarse las diferentes variables entre el sujeto y el estrés. De esta manera De Dios Duarte, Varela, Braschi y Sánchez (2017) refieren los componentes personales, los cuales son "el apoyo social, el estilo de afrontamiento, la respuesta biológica, variables relacionadas con la toma de decisiones y variables de personalidad", todo esto es reafirmado por Gaibor, Liger, Safla, Fernández y Cholota en 2018, expresan que el ser humano puede llegar a estresarse por diferentes factores, esto dependerá en gran medida a la personalidad del sujeto, el suceso y como está su estado mental al momento de lo ocurrido. 


\section{Materiales y métodos}

La investigación se realizó con un enfoque cuantitativo siendo los resultados medibles y cuantificados, de tipo descriptivo, mediante el método inductivo-deductivo.

La población fue constituida por los militares en servicio pasivo de la Asociación de Militares en Servicio Pasivo de las FF.AA. "Veteranos de Guerra y Anexos", de Santo Domingo de los Tsáchilas, la cual posee alrededor de 107 miembros. La muestra es de tipo intencional: conformado por 87 militares en servicio pasivo, que oscilaron entre las edades de 44 a 60 y mayores de 60. Participaron miembros del sexo masculino. Se tomó en cuenta a los miembros que están en constante contacto con el resto de los miembros de la asociación, no se incluyó a los sujetos que necesitan cuidados especiales y las parejas de los militares que han fallecido.

\section{Instrumentos:}

Se empleó el inventario sobre estrés de Melgosa, 1994, el mismo que consta de 96 ítems que buscan explorar el nivel de estrés experimentado:

- Estilo de vida
- Ambiente

- Síntomas

- Trabajo/Ocupación

- Relaciones

- Personalidad

La escala brinda cuatro opciones de respuesta: "nunca", "casi nunca", "frecuentemente" y "casi siempre", cada uno tiene una valoración del 0 al 4 según sea el caso, el inventario permite determinar el nivel de estrés según la puntuación que se obtenga, a mayor puntuación, mayor será el nivel de estrés:

- Zona 1: El nivel de estrés es peligrosamente pobre. Necesita un poco de chispa en su vida para alcanzar los logros que esperan de usted.

- Zona 2: Disfruta usted de un nivel bajo de estrés. Puede deberse a su naturaleza apacible, gozando una situación saludable. Pese a todo, podría estar rindiendo por debajo de su capacidad, sería necesario imponerse retos.

- Zona 3: Esta la zona normal de estrés. La mayoría de las personas se encuentran en esta zona, a veces hay tensiones, $y$ otras veces momentos de 
relajación. Si la puntuación está cerca del límite superior, como medida de prudencia, considérese al menos, parcialmente, en la zona 4.

- Zona 4: El estrés en esta zona se considera elevado. Usted está recibiendo un aviso claro y contundente de peligro. Adopte una actitud positiva y procure ser amable con todos.
- Zona 5: Esta zona se considera peligrosa. Si usted alcanza una puntuación mayor a 144 , usted se encuentra en un grupo reducido de personas muy estresadas y con múltiples problemas que requieren atención inmediata. Si le es posible, cambie de actividad durante unos días y aplique tantas técnicas y estrategias antiestrés como sea posible.

Figura 1. Inventario sobre estrés de Melgosa.

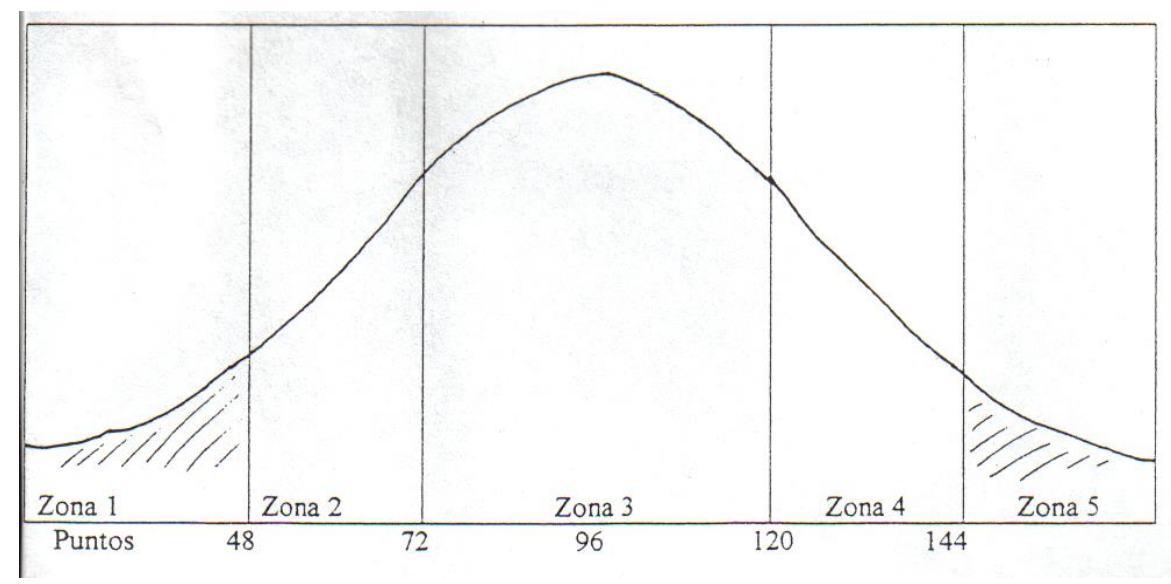

Fuente: (Melgosa, 1994)

\section{Resultados}

Gráfico 1. Nivel de estrés en militares en servicio pasivo mayores de 60 años sin trabajo.

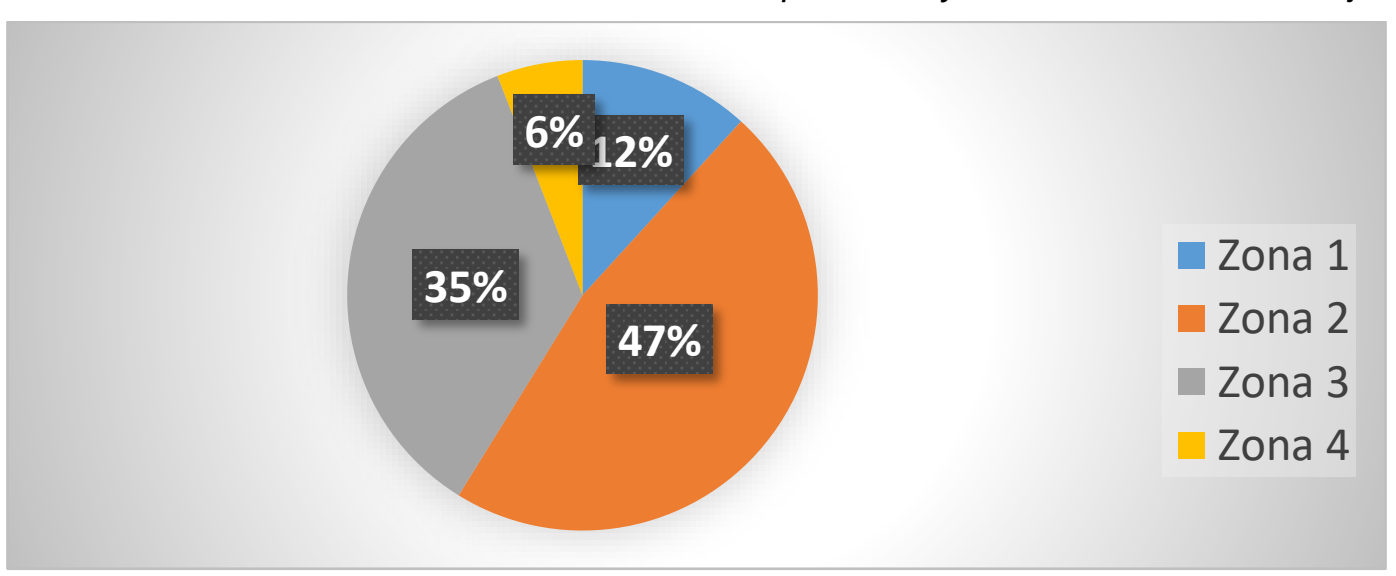

Fuente: Elaboración propia. 
A través del gráfico 1 se puede evidenciar que el $47 \%$ de la población de más de 60 años de edad sin trabajo se encuentran en la zona 2 que demuestra un nivel bajo de estrés, mientras que el $35 \%$ se encuentra en la zona 3 con un nivel normal de estrés, el $12 \%$ se encuentra en la zona 1 de estrés bajo y que el $6 \%$ en la zona 4 presentando un nivel de estrés de considerado elevado.

Gráfico 2. Nivel de estrés en militares en servicio pasivo mayores de 60 años con trabajo.

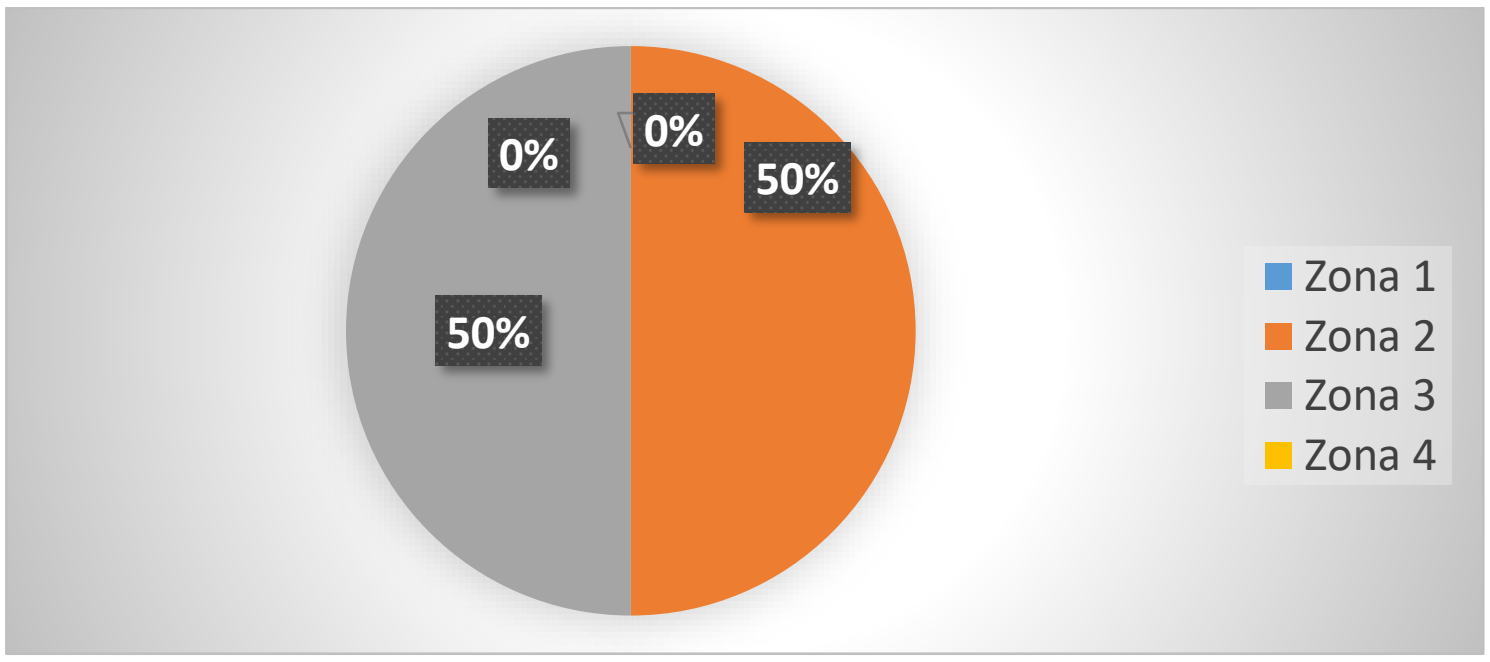

Fuente: Elaboración propia.

A través del gráfico 2 se identifica que el $50 \%$ de la población de más de 60 años de edad con trabajo se encuentran en la zona 2 que demuestra un nivel bajo de estrés, mientras que el $50 \%$ se encuentra en la zona 3 con un nivel normal de estrés.

Gráfico 3. Nivel de estrés en militares en servicio pasivo menores de 60 años sin trabajo.

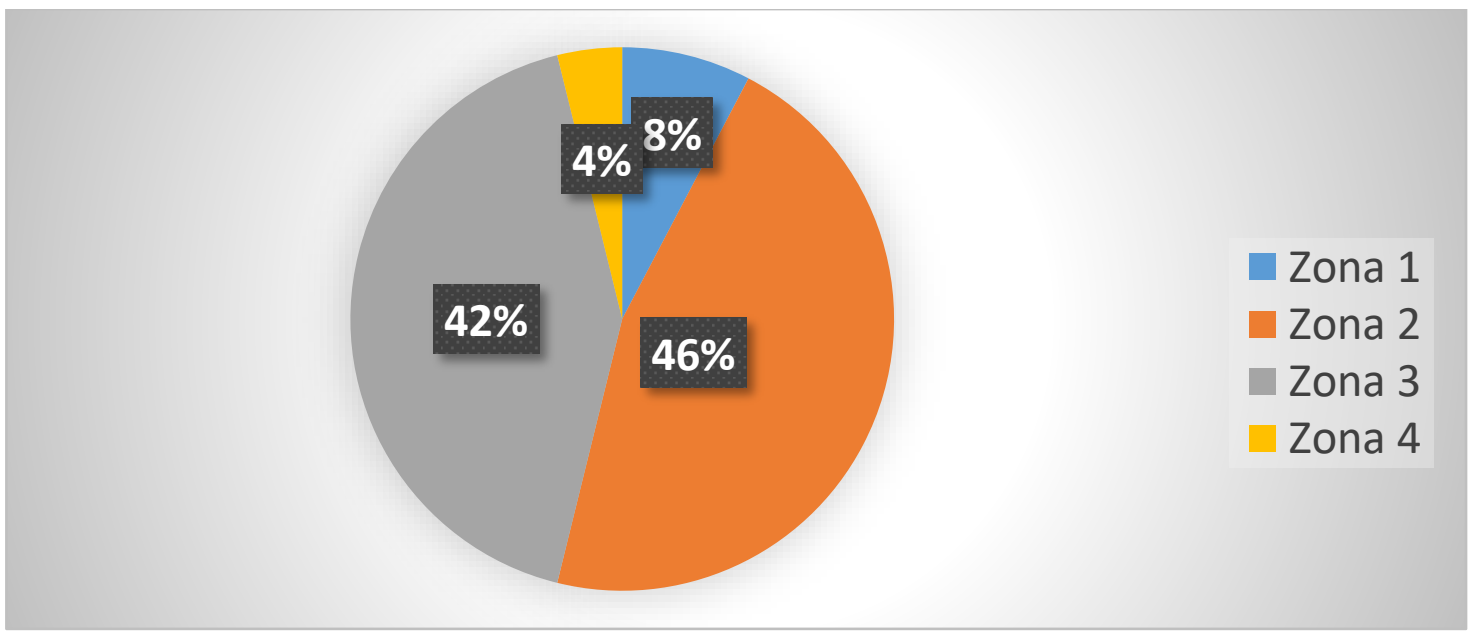

Fuente: Elaboración propia. 
A través del gráfico 3 se muestra que

el $46 \%$ de la población menor de 60 años de edad sin trabajo se encuentran en la zona 2 que demuestra un nivel bajo de estrés, mientras que el $42 \%$ se encuentra en la zona 3 con un nivel normal de estrés, el $8 \%$ se encuentra en la zona 1 de estrés bajo y que el $4 \%$ en la zona 4 presentando un nivel de estrés de considerado elevado.

Gráfico 4. Nivel de estrés en militares en servicio pasivo menores de 60 años con trabajo.

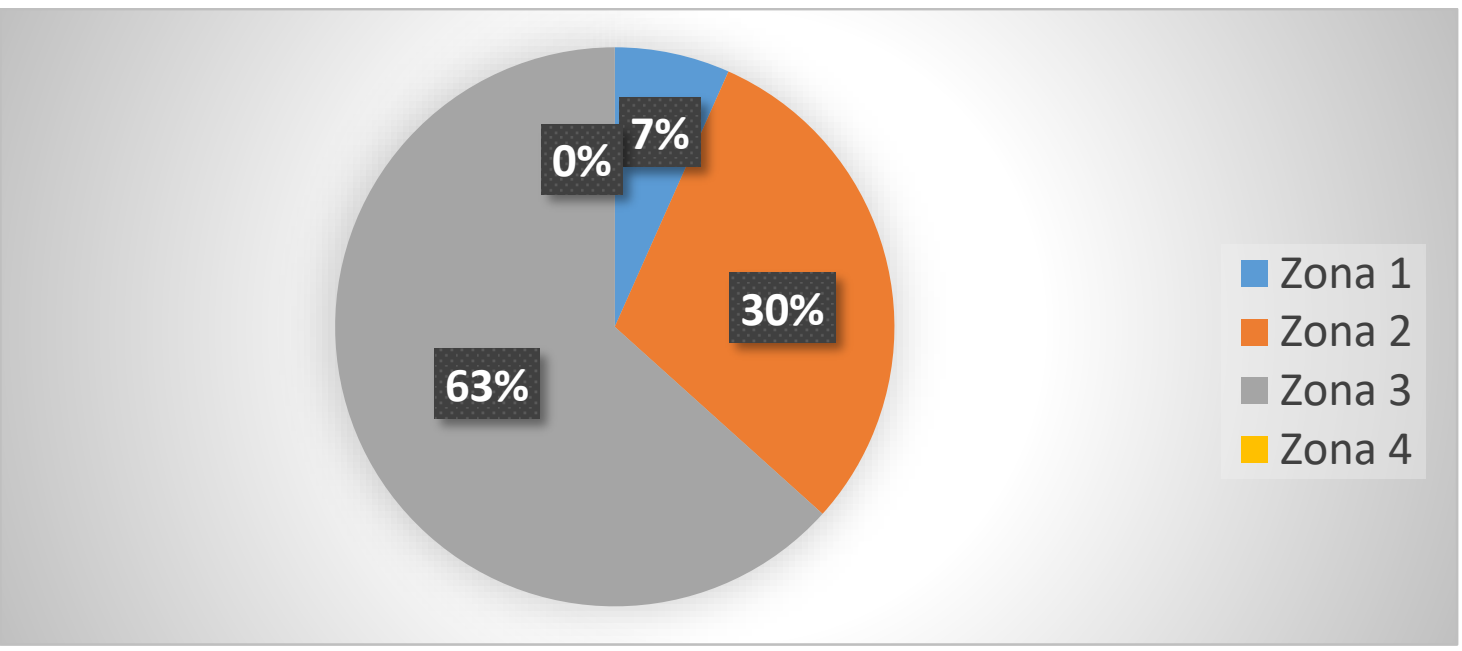

Fuente: Elaboración propia.

A través del gráfico 4 se puede evidenciar que el $63 \%$ de la población menor de 60 años de edad con trabajo se encuentran en la zona 3 que demuestra un nivel bajo de estrés, mientras que el $30 \%$ se encuentra en la zona 2 con un nivel normal de estrés, el $7 \%$ se encuentra en la zona 1 de estrés bajo.

\section{Discusión}

En la presente investigación se buscó determinar el nivel de estrés que ocasionan los distintos factores psicosociales en militares en servicio pasivo de la Asociación de Militares en Servicio Pasivo de las FF.AA. "Veteranos de Guerra y Anexos" de Santo Domingo de los Tsáchilas.

Se identificó a 87 militares que corresponde al $81 \%$ de la población total de sexo masculino que cumplen los criterios de inclusión y exclusión de los cuales 56 de ellos, siendo el $64 \%$ la población menor de 60 años y 31 de ellos son el $36 \%$ de la población mayor a 60 años.

Se encontró en la población que tiene más de 60 años de edad, el $48 \%$ se encuentra en la zona 2 que 
representa un nivel bajo de estrés, el42\% se ubica en la zona 3 siendo un nivel de estrés normal, a comparación de los sujetos menores a 60 años de edad, el 54\% está en la zona 3 con nivel normal de estrés y el $37 \%$ en la zona 2 con un nivel bajo de estrés. Analizando los resultados entre ambos rangos de edad se evidencia que la población menor de 60 años presenta un nivel de estrés normal correspondiente a la zona 3, más que en la población mayor de 60 años de edad.

En los gráficos 1 y 3 se muestra a la población sin trabajo donde predomina la zona 2 en el nivel bajo de estrés sobre la zona 3 en el nivel normal de estrés. A diferencia de las gráficas 2 y 4 de la población con trabajo sobre sale la zona 3 correspondiente al nivel normal de estrés a diferencia de la zona 2 del nivel bajo de estrés. El total de la población presento un porcentaje poco significativo en la zona 4 y zona 1 del nivel de estrés.

\section{Conclusiones}

Como se pudo comprobar en la teoría en cuanto al estrés, existen diversos factores que puede favorecer al desarrollo del estrés en todas las profesiones, sin embargo, la profesión de los militares tiene un ambiente estresante, se encuentran expuestos a estrés constante ante diferentes factores, que en su mayoría son propias del contexto laboral y la organización cultural de las fuerzas armadas. Teniendo en cuenta que los sujetos que formaron parte de esta investigación tuvieron participación en la guerra del CENEPA, deberían de haber recibido ayuda psicológica, por el mismo hecho de haber estado en circunstancias extremadamente estresantes.

Con el contenido de este documento podemos constatar que los militares en servicio activo están ante diferentes factores que puede ir atribuyendo a un desarrollo del estrés, están en un ambiente que puede producir afectaciones psicológicas, que pudieran afectar su nivel social y laboral. Además de esto, después de varios años de servir como militares sufren un agotamiento tanto físico como psicológico, los sujetos se encontraban ante la presión de un horario establecido de gran rigurosidad, ser soldados en conflictos armados, vivir lejos de sus 
familias, tener que obedecer estrictamente a las ordenes dados por sus superiores, etc.

En la población mayor de 60 años de edad podemos destacar que se encuentran en una zona 2 según el inventario de Melgosa, lo que nos indica que su nivel de estrés es bajo, pero por otro lado los menores de 60 años de edad se encuentran en una zona 3 que representaría que están con un estrés normal. En cuanto al análisis estadístico podremos decir que en mayor medida los militares en servicio pasivo presentan niveles de estrés bajos y normales, existiendo pequeños grupos que presentan niveles de estrés elevados que deberían ser tratados, evaluados y tratados por un profesional de la salud mental.

\section{Recomendaciones}

Como defensores de la patria, que han puesto en riesgo sus factores sociales, físicos, psicológicos, pero sobre todo su propia vida, deberían de tener mayor seguimiento durante el servicio activo como en el servicio pasivo, ayudando a que no pueda presentarse alguna afectación a nivel psicológica o emocional en su nueva vida fuera del ejército. Lo que se estaría buscando no es que continúen siendo líderes militares, sino más bien verdaderos seres humanos.

Las fuerzas armadas deben contar con gran variedad de recursos en cuanto a la salud mental de sus militares, con esto nos referimos que debe existir más de un método que pueda facilitar la resolución de conflictos psíquicos, según las necesidades y creencias de los sujetos, con el propósito de que una vez finalizada su vida laboral pueda integrarse a su nueva realidad con facilidad.

Los militares en servicio pasivo se enfrentan a diversos conflictos entre ellos estaría el desempleo, buscar nuevas oportunidades de empleo, estar acostumbrados a sus nuevas libertades, reemplazar su antigua vida de militar con nuevas actividades, según lo observado existe un patrón en sus relaciones interpersonales, son sujetos que tienen un microsistema que se rige entre sus pares. 


\section{Bibliografía}

Acurio, A. y Morejón, M. (2017). Inteligencia emocional y estrés laboral en militares de la brigada de caballería blindada no 11 "galápagos" de Riobamba, período marzo agosto 2016. [Tesis de pregrado, Repositorio Digital UNACH].

http://dspace.unach.edu.ec/h andle/51000/4073

De Dios Duarte, J., Varela, I., Braschi, L. y Sánchez, E. (2017). Estrés en estudiantes de enfermería. Educación Médica Superior, 31(3), 110123.

http://scielo.sld.cu/scielo.php? script=sci_arttext\&pid=S0864 $-21412017000300013$

Espinoza, A., Pernas, I. y Gonzales, R. (2018). Consideraciones teórico metodológicas y prácticas acerca del estrés. Scielo 18(3). http://scielo.sld.cu/scielo.php? script=sci_arttext\&pid=S1727 -81202018000300697

Flores, X. y Serrano, A. (2019). Incidencia de las demandas del trabajo en el nivel de estrés del personal del Depósito Conjunto de Municiones "El Corazón" (Depcor). [Tesis de maestría, UASB-Digital]. http://hdl.handle.net/10644/70 57

Domjan, M. (2016). Principios de aprendizaje y conducta. (J.
Fernández, trad.). CENGAGE Learning.

Personal, reclutamiento y movilización. (1987, juniojulio). La cohesión: el elemento humano en el combate (segunda parte). [boletín n. ${ }^{\circ}$ 202]. Universidad de Defensa Nacional. http://www.bibliotecavirtualdef ensa.es/BVMDefensa/i18n/ca talogo_imagenes/grupo.cmd? path $=71706$

Ospina, A. (2016). Síntomas, niveles de estrés y estrategias de afrontamiento en una muestra de estudiantes masculinos y femeninos de una institución de educación superior militar: análisis comparativo. [Tesis de maestría, Universidad católica de Colombia]. Repositorio Institucional Universidad Católica de Colombia.

http://hdl.handle.net/10983/31 61

Gaibor, J., Liger, T., Safla, j., Fernández, M. y Cholota, L. (2018). El estrés en las fuerzas armadas: la situación de estrés en los aspirantes a soldados en la ESFORSE, promoción 2015-2017. Revista de Ciencias de Seguridad y Defensa, 3(3), 50-60.

https://biblioguias.ucm.es/estil o-apaseptima/articulo_revista

Loaiza, O., \& Posada, J. L. (2016). Psicología militar: 
Conceptualización e investigaciones

contemporáneas. PSIENCIA. Revista Latinoamericana de Ciencia Psicológica, 8(2), 120.

https://www.redalyc.org/articu lo.oa? id=333147069006

Melgosa J. (1994). ¡Sin estrés!. Editorial Safeliz. ISBN: 847208-101-X

Norma, S. (2017). Factores asociados al estrés laboral en el personal de Enfermería del área de emergencia y unidad de cuidados intensivos de un hospital de la ciudad de Guayaquil. [Tesis de pregrado, Universidad Católica de Santiago de Guayaquil]. Repositorio Digital UCSG. http://repositorio.ucsg.edu.ec/ handle/3317/8987

Rodrigo, L. (2015). Persona, sexo y género. Los significados de la categoría «género» y el sistema «sexo/género» según Karol Wojtyła. Open Insight, (7)12, 143-168.

Rodríguez, J. y Arce, R. (2016). Militares desplegados en misiones internacionales: percepción del estrés y síntomas asociados. Sanidad Militar, $\quad$ 72(1). 15-24 http://scielo.isciii.es/scielo.ph p?script=sci_arttext\&pid=S18 87-

$85712016000100003 \&$ Ing $=\mathrm{es}$ \&tlng=es
Ruiz, M. (2017). Revisión literaria de protocolos de atención psicológica en estrés postraumático aplicados a población militar. [Tesis de pregrado, Universidad cooperativa de Colombia]. Repositorio Institucional UCC. https://repository.ucc.edu.co/ handle/20.500.12494/13956

Vela, P. (2018). Estrés laboral, tipos de personalidad y estrategias de afrontamiento: estudio realizado en el "Colegio Militar Eloy Alfaro" de la ciudad de Quito durante el primer quimestre del año lectivo 2017-2018. [Tesis de pregrado, Pontificia Universidad Católica del Ecuador Facultad de medicina]. Repositorio de Tesis de Grado y Postgrado. http://repositorio.puce.edu.ec/ handle/22000/14756 